\title{
Multifunctional Metasurfaces Based on the "Merging" Concept and Anisotropic Single-Structure Meta-Atoms
}

\author{
Shiwei Tang ${ }^{1, *} \mathbb{D}$, Tong Cai ${ }^{2}$, He-Xiu Xu ${ }^{2}$, Qiong $\mathrm{He}^{3}$, Shulin Sun ${ }^{4}$ and Lei Zhou ${ }^{3, *}$ \\ 1 Department of Physics, Faculty of Science, Ningbo University, Ningbo 315211, China \\ 2 Air and Missile Defend College, Air force Engineering University, Xi'an 710051, China; \\ caitong326@sina.cn (T.C.); hxxuellen@gmail.com (H.-X.X.) \\ 3 State Key Laboratory of Surface Physics, Key Laboratory of Micro and Nano Photonic Structures (Ministry \\ of Education), Collaborative Innovation Center of Advanced Microstructures, and Physics Department of \\ Fudan University, Shanghai 200433, China; qionghe@fudan.edu.cn \\ 4 Shanghai Engineering Research Center of Ultra-Precision Optical Manufacturing, Green Photonics and \\ Department of Optical Science and Engineering, Fudan University, Shanghai 200433, China; \\ sls@fudan.edu.cn \\ * Correspondence: tsw@fudan.edu.cn (S.T.); phzhou@fudan.edu.cn (L.Z.); \\ Tel.: +86-180-067-06998 (S.T.); +86-021-5566-5236 (L.Z.)
}

Received: 7 March 2018; Accepted: 2 April 2018; Published: 4 April 2018

\begin{abstract}
Metasurfaces offer great opportunities to control electromagnetic (EM) waves, attracting intensive attention in science and engineering communities. Recently, many efforts were devoted to multifunctional metasurfaces integrating different functionalities into single flat devices. In this article, we present a concise review on the development of multifunctional metasurfaces, focusing on the design strategies proposed and functional devices realized. We first briefly review the early efforts on designing such systems, which simply combine multiple meta-structures with distinct functionalities to form multifunctional devices. To overcome the low-efficiency and functionality cross-talking issues, a new strategy was proposed, in which the meta-atoms are carefully designed single structures exhibiting polarization-controlled transmission/reflection amplitude/phase responses. Based on this new scheme, various types of multifunctional devices were realized in different frequency domains, which exhibit diversified functionalities (e.g., focusing, deflection, surface wave conversion, multi-beam emissions, etc.), for both pure-reflection and pure-transmission geometries or even in the full EM space. We conclude this review by presenting our perspectives on this fast-developing new sub-field, hoping to stimulate new research outputs that are useful in future applications.
\end{abstract}

Keywords: metasurface; multifunctional device; metamaterial; meta-atom

\section{Introduction}

Facing the increasing demands on data-storage capacity and information processing speed in modern science and technology, electromagnetic (EM) integration plays a more and more important role, which has intrigued intensive attention with remarkable applications. An ultimate goal pursued by scientists and engineers along this development is to make miniaturized devices as small as possible, yet equipped with powerful functionalities as many as possible. However, available efforts based on conventional materials suffer from the issues of device thickness, low efficiency, and restricted functionalities, caused by the fact that natural materials only exhibit electric responses with small variation range of permittivity $\varepsilon$ and, thus, they only have limited capabilities on manipulating EM waves. 
Metamaterials (MTMs) [1,2], consisting of deep-subwavelength-sized EM microstructures (e.g., meta-atoms) arranged in periodic or non-periodic orders, have drawn much attention recently. Through tailoring the microstructures of meta-atoms, MTMs can, in principle, exhibit arbitrary values of permittivity $\varepsilon$ and permeability $\mu$, which offers MTMs extraordinarily strong capabilities to control EM waves. Many fascinating wave-manipulation effects have been demonstrated based on MTMs, such as negative refraction [3,4], super-resolution imaging [5,6], cloaking [7-9], polarization-control [10-13], perfect light absorption [14], and transparency $[15,16]$, and unusual wave-control effects realized by zero-index MTMs [17,18]. Attempts have also been made to achieve multifunctional EM devices based on MTMs. However, the realized devices typically exhibit bulky sizes and low efficiencies, since MTMs are three-dimensional (3D) materials composed by resonant metallic structures which can easily absorb EM waves. Moreover, such 3D devices require complex fabrication processes, adding more disadvantages for EM integration $[19,20]$.

Metasurfaces, ultrathin MTM layers constructed by planar meta-atoms of pre-determined EM responses arranged in specific two-dimensional (2D) orders, can largely overcome the difficulties faced by MTMs. Tuning the EM responses of meta-atoms to realize certain transmission/reflection phase distributions on the metasurfaces, one can use these ultra-thin devices to efficiently reshape the wave-fronts of incident EM beams based on Huygens' principle, achieving unusual effects, including anomalous beam bending based on generalized Snell's law [21-29], propagating wave to surface waves conversion [30-32], polarization-control [33-39], focusing [40-42], holograms [43-45], flat-lens imaging [46-50], tunable devices [51-53], and photonic spin-Hall effect [54-56], etc. Typically, these devices are flat, much thinner than the wavelength, and exhibit much higher efficiencies than their bulky MTM counterparts, all being highly favorable for integration-optics applications. These attractive properties make metasurfaces the best candidates to construct multifunctional EM devices. Indeed, many efforts have recently been devoted to designing multifunctional optical devices based on metasurfaces [57-87], typically using polarization or frequency of the incident light as an external knob to control the functionality exhibited by the devices. The proposed/demonstrated devices are usually equipped with functionalities combining two or more from those demonstrated before on single-function metasurfaces, such as beam-bending, focusing, hologram, surface-wave conversion, and directive beaming, etc.

In this paper, we present a concise review on this fast developing sub-field, focusing on the working principles and practical realizations of multi-functional metasurface-based meta-devices. We particularly emphasize the importance of designing appropriate meta-atoms in this field, since the remaining tasks are merely engineering optimizations after high-performances meta-atoms are found. This review is organized as follows. We first briefly summarize in Section 2 a class of multifunctional meta-devices based on the "merging" concept. Having understood the merits and disadvantages of the "merging" scheme, we then introduce another class of multifunctional metasurfaces based on single-structure meta-atoms exhibiting polarization-controlled transmission/reflection phase responses. The realized meta-devices include those exhibiting similar wave-control functionalities for two polarizations (Section 3.1), those integrating different functionalities with very high efficiencies in reflection geometry (Section 3.2) and in transmission geometry (Section 3.3), and those utilizing the full EM space to control EM waves with different capabilities (Section 3.4). We finally conclude this review and present our perspectives in the last section.

\section{Multifunctional Meta-Devices Based on the "Merging" Concept}

A simple scheme developed in early years utilized the so-called "merged" meta-structures to design multifunctional metasurfaces. In such a scheme, people first design individual metasurfaces exhibiting their own functions and then construct a multifunctional device simply through merging the two structures together. Below we present several examples to illustrate how the scheme works.

Figure 1a presents an optical bifunctional metasurface that can realize a hologram image or a vortex beam, depending on the helicity of excitation light [74]. To achieve their end, the authors 
first design two individual metasurfaces (both utilizing the metal-bar structure as basic meta-atoms) which can realize one of the needed functionalities when they are shined by incident light taking circular polarizations (CP) with different helicities (see Figure 1a). The desired phase profiles on two metasurfaces are created by the Pancharatnam-Berry $(\mathrm{PB})$ principle $[54,88,89]$ through rotating the metallic bars at different positions by appropriate angles. Since the two metasurfaces exhibit identical periodic structures and there are enough open spaces between metallic bars, the authors then merge two metasurfaces together to obtain the final design in which all metallic bars do not touch with each other. Such a device was finally fabricated out and experimentally characterized, showing nice bifunctional performances (Figure 1a). However, the working efficiency of the device is quite low, which is found to be around 9\% [74].

(a)

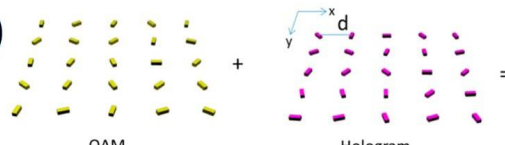

OAM

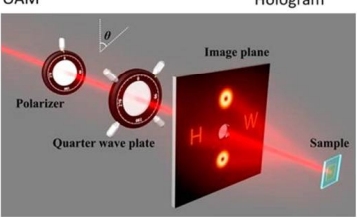

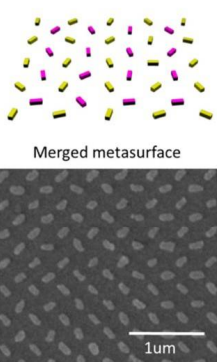

(c)

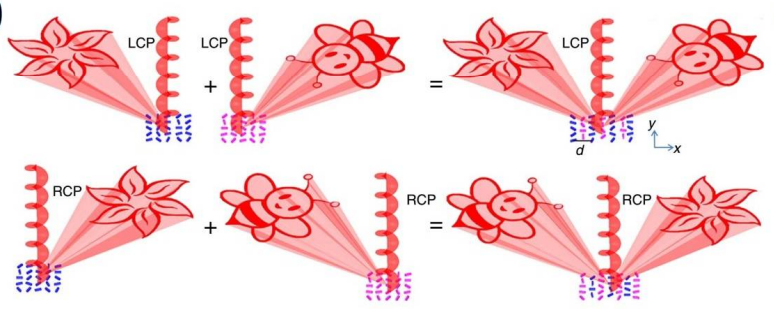

(b)

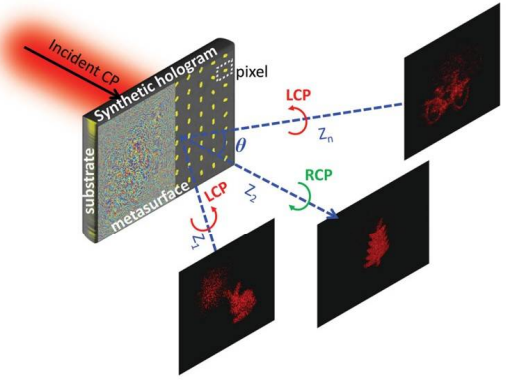

(d)

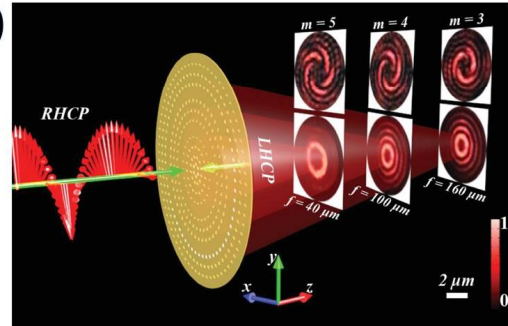

Figure 1. Multifunctional devices designed with merged structures. (a) Design strategy, sample picture, and experimental characterizations of a multifunctional metasurface than can generate holographic images or a vortex beam depending on the helicity of incident circularly polarized light. Reproduced from Ref. [74] with permission. (b) A metasurface that can reconstruct different images to different polarization channels. Reproduced from [64] with permission. (c) A metasurface that can generate multiple hologram images as shined by circularly polarized light with different helicity. Reproduced from [63] with permission. (d) A metasurface that can generate optical vortices with distinct topological charges at different longitudinal focal planes. Reproduced from [58] with permission. LCP, left CP; $\mathrm{RCP}$, right CP; OAM, orbital angular momentum; LHCP, left-handed circularly polarized ; RHCP, right-handed circularly polarized.

Such a "merging" concept has been straightforwardly applied to realize many other multifunctional meta-devices [58,61-65]. Figure 1c presents a reflective bifunctional meta-device which can form a holographic image of a 'flower' or a 'bee' as the helicity of incident light changes from left $C P(\mathrm{LCP})$ to right $\mathrm{CP}(\mathrm{RCP})$ [63]. In Figure 1b, a new type of meta-hologram device is introduced, which can exhibit various hologram patterns depending on the helicity the incident light [64]. Figure $1 \mathrm{~d}$ shows a meta-device which can control the polarization state (i.e., spin angular momentum) and the position of the focal planes by manipulating the helicity of the incident light. [58].

In reviewing these meta-devices based on the "merging" concept, we find that the proposed design strategy is physically transparent and easy to implement. However, to make the "merging" process work, the adopted meta-atoms must be very simple structures (say, metal bar) to avoid metallic overlapping. Unfortunately, these meta-atoms typically do not satisfy the $100 \%$-efficiency criterion established for PB metasurfaces [54] and, thus, one type of meta-atoms can generate background noise in addition to the desired functionalities. As a result, such meta-devices typically suffer from issues of 
low operating efficiencies and functionality cross-talking, except [63] where the issue was partially solved by seeking a high-efficiency PB meta-atom in the reflection geometry.

\section{Multifunctional Metasurfaces Based on Anisotropic Single-Structure Meta-Atoms for Two Polarizations}

Having understood the key issues in the "merging" concept, people propose a new strategy to design multifunctional metasurfaces, i.e., using single structures as the basic meta-atoms to design the final device instead of merging two separately determined ones. In what follows, we first discuss the basic concept, and then introduce several meta-devices realized with such a strategy, classified into four sub-categories as detailed below.

Suppose that the adopted meta-atoms possess mirror symmetries, we can then describe the EM response for a meta-atom located at a position $(x, y)$ on a metasurface by two diagonal Jones's matrices:

$$
\mathbf{R}=\left(\begin{array}{cc}
r_{x x}(x, y) & 0 \\
0 & r_{y y}(x, y)
\end{array}\right)
$$

and:

$$
\mathbf{T}=\left(\begin{array}{cc}
t_{x x}(x, y) & 0 \\
0 & t_{y y}(x, y)
\end{array}\right)
$$

where $r_{x x}, r_{y y}, t_{x x}, t_{y y}$ denote the reflection/transmission coefficients of the meat-atom (periodically repeated to form a periodic metasurface), respectively. In order to make the devices exhibit working efficiencies as high as possible, ideally one requires the designed meta-atoms to be either perfectly reflective:

$$
\mathbf{T}=0 ;\left|r_{i i}\right|=1
$$

or perfectly transparent:

$$
\mathbf{R}=0 ;\left|t_{i i}\right|=1 .
$$

Once such conditions are satisfied meaning that all meta-atoms are of highest working efficiencies, people then adjust the geometrical parameters of each meta-atom to make the whole metasurface exhibit desired polarization-dependent phase profiles (i.e., $\varphi_{x x}(x, y)$ and $\left.\varphi_{y y}(x, y)\right)$ to achieve certain wave-manipulation functionalities. A typical example is schematically shown in Figure 2, for which the wanted bi-functionalities are focusing and beam bending, respectively. To achieve this end, the final phase distributions on the metasurface should be:

$$
\varphi_{y y}(x, y)=k_{0}\left(\sqrt{F^{2}+x^{2}+y^{2}}-F\right)
$$

with $F$ being the focal length and $k_{0}$ being the free-space wave-vector, and:

$$
\varphi_{x x}(x, y)=C_{1}+\xi \cdot x
$$

with $\xi$ being the phase gradient responsible for the anomalous reflection angle and $C_{1}$ being an a constant. We note that a conversion from propagating wave (PW) to surface wave (SW) can happen if $\xi>k_{0}$.

We emphasize that the high-efficiency conditions Equations (1) and (2) can only be approximately satisfied since losses inevitably exist, especially at high frequencies. Moreover, the achieved functionalities are not limited to the two described above. If different wave-manipulation functionalities are desired, one may easily replace Equations (5) and (6) by the phase distributions related to those functionalities. 

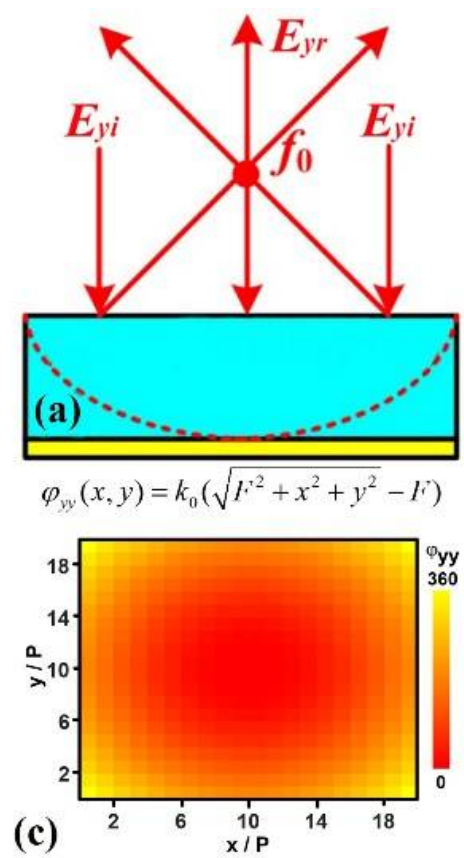

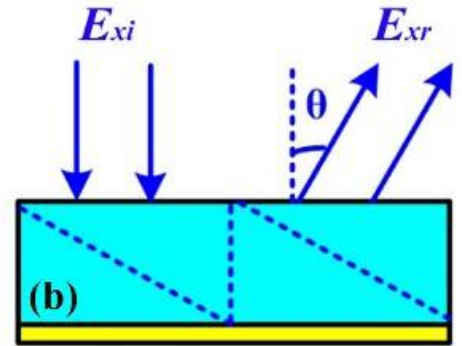

$\varphi_{x x}(x, y)=C_{1}+\xi \bullet x$

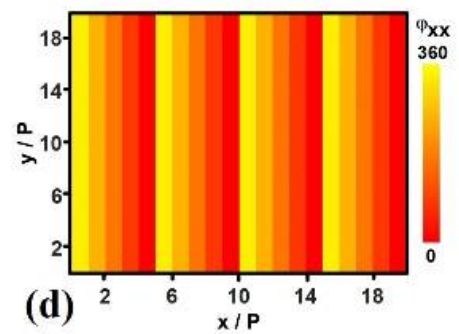

Figure 2. Schematics of a bi-functional metasurface (blue) with metal ground plane (yellow) which can achieve (a) focusing functionality for $y$-polarized incident light and (b) anomalous-reflection functionality for $x$-polarized incident light. The phase distributions of the metasurface are depicted in (c) for $y$-polarization excitation and in (d) for $x$-polarization excitation. Reproduced from [90] with permission.

\subsection{Multifunctional Metasurfaces Exhibiting Similar Functionalities}

Historically, multifunctional metasurfaces designed by single-structure meta-atoms were first realized in reflection geometry, because high-efficiency reflective meta-atoms are much easier to find than their transmissive counterparts. A commonly-used reflective meta-atom is the so-called metal-insulator-metal (MIM) structure, which consists of a metallic planar resonator and a continuous metal sheet separated by a dielectric spacer (see Figure 3a). Such an MIM structure was widely used to design high-efficiency reflective metasurfaces at frequencies ranging from microwave to visible $[22,23,44,72,79,91]$, simply because its metallic ground plane can help reflect all incoming waves back (thus, Equation (1) can be easily satisfied) while the phase of reflected wave can undergo a continuous $-180^{\circ}$ to $180^{\circ}$ variation as frequency passes through a resonance inside the "meta-atom". Such a resonance is usually called "magnetic resonance" [14] or "gap-plasmon resonance" [22,72], and its properties are carefully analyzed in [91,92]. Therefore, tuning the geometrical parameters of such a meta-atom can efficiently modify its two reflection phases $\left(\varphi_{x x}\right.$ and $\left.\varphi_{y y}\right)$ (see Figure $3 b$ ), which then offers enough freedoms to design multifunctional meta-devices.

Figure $3 \mathrm{~d}$ depicts a bifunctional meta-device working at telecom wavelengths, which can excite surface plasmon polaritons (SPPs) to flow along two orthogonal directions when the device is shined by external light with polarizations along $x$ or $y$ direction [72]. The fabricated device consists of 3 $\times 3$ MIM meta-atoms, with geometrical parameters carefully adjusted such that both $\varphi_{x x}(x, y)$ and $\varphi_{y y}(x, y)$ satisfy Equation (6) with $\xi>k_{0}$. Experimental characterizations reveal that the fabricated device can indeed exhibit the desired bi-functionalities (see Figure 3a). Based the same concept, other bi-functional meta-devices were realized based on MIM meta-atoms with patches replaced by other type of planar resonators, to achieve the polarization-dependent anomalous reflection (beam splitting) (Figure 3c) [79] and polarization-controlled dual-image hologram (Figure 3e) [59]. Two common features of these meta-devices are worth mentioning: the realized functionalities are identical 
or similar, and the working efficiencies are not very high, partially caused by the metallic losses at optical frequencies.
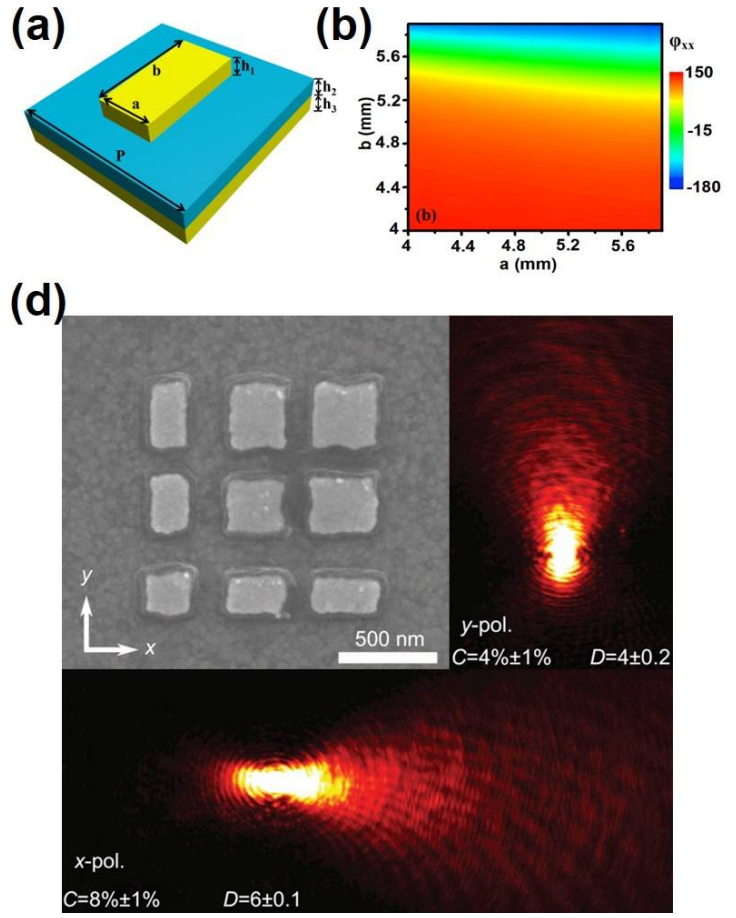

(c)

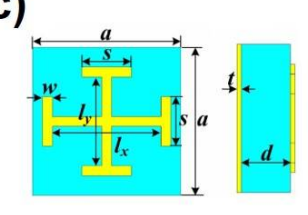

(e)
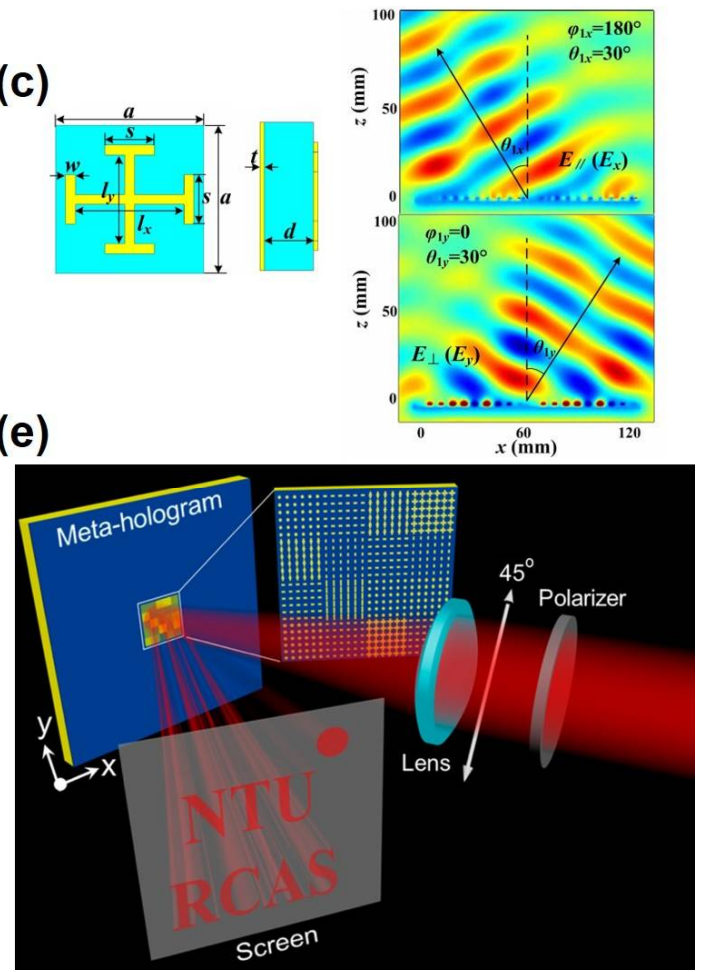

Figure 3. (a) Schematics of an MIM meta-atom consisting of a metallic patch resonator and a metal ground plane (yellow) separated by a dielectric spacer (blue). (b) Tuning the reflection phase $\varphi_{x x}$ of a microwave MIM meta-atom by varying a and b. Reproduced from [93] with permission. (c) A polarization beam splitter made by bifunctional gradient metasurface constructed with MIM meta-atoms consisting of metal-cross planar resonators and metal ground plane (yellow) separated by a dielectric spacer (blue). Reproduced from [79] with permission. (d) Experimental characterizations on a unidirectional polarization-controlled SPP (red) coupler at telecom wavelength. Reproduced from [72] with permission. (e) A bi-functional meta-hologram device consisting of metal pattern and metal ground plane (yellow) separated by a dielectric spacer (blue) that can generate different hologram image depending on the incident linear polarization. Reproduced from [59] with permission.

\subsection{Multifunctional Reflective Metasurfaces Combining Distinct Functionalities}

The proposed design strategy is so general that it can also be used to realize multifunctional metasurfaces exhibiting distinct functionalities. For example, the first reflective multifunctional meta-device possessing two distinct functionalities (focusing and PW-to-SW conversion) was demonstrated in [93] in the microwave regime (see Figure 4a,b). Taking meta-atoms the same as shown in Figure 3a, the authors successfully designed a reflective bi-functional metasurface which exhibits two phase distributions satisfying Equations (5) and (6). Shining a $y$-polarized microwave at frequency 9.3 GHz normally onto the sample, the authors measured the $\operatorname{Re}[\vec{E}]$ distributions on two orthogonal planes. Results displayed in Figure $4 \mathrm{c}$ indicate clearly that the incoming plane wave has been focused to a focal point. To demonstrate the PW-to-SW conversion effect for the $\vec{E} \| \hat{x}$ polarization, the authors purposely fabricated a mushroom structure supporting eigen spoof SPP [30,32] and then put it at the right side of the metasurface to guide out the driven SW (see inset to Figure 4d). Shining the metasurface by an $x$-polarized microwave $(9.3 \mathrm{GHz})$, the authors used a near-field scanning technique to map out the $\operatorname{Re}\left[\vec{E}_{z}\right]$ field pattern (see Figure $4 \mathrm{~d}$ ). The measured field pattern represents a very 
well-defined spoof SPP with $k_{s p p}=206.5 \mathrm{~m}^{-1}$, in good agreement with the theoretically-calculated value $\left(k_{s p p}=209.4 \mathrm{~m}^{-1}\right)$. For both functionalities, the experimentally-estimated working efficiencies are higher than $90 \%$, which is not surprising since metallic losses are negligible at microwave frequencies. Such a concept was successfully extended to realize the same type of bifunctional meta-device, but working at optical wavelengths (Figure 4e) [94], which is more close to realistic integration-optics applications.
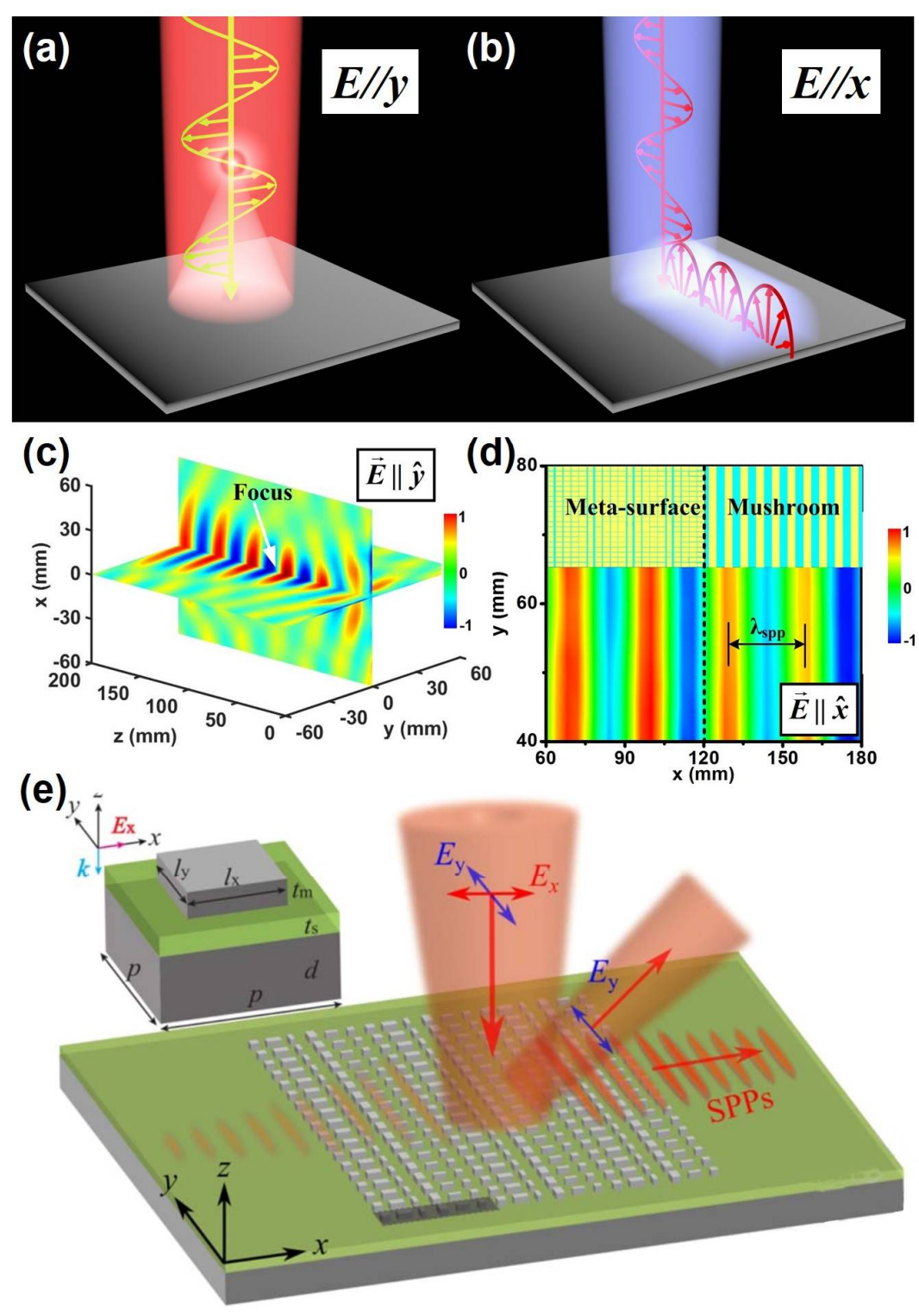

Figure 4. A reflective bifunctional metasurfaces that behaves as (a) a focusing lens and (b) a PW-to-SW (propagating wave to surface wave) convertor when excited by incident waves with polarizations $E / / y$ (red light) and $E / / x$ (blue light), respectively. (c) Measured $\operatorname{Re}[\vec{E}]$ distributions on both $x_{\mathrm{o} z}$ and yoz planes as the metasurface is illuminated by a normally incident $y$-polarized plane wave. (d) Measured $\operatorname{Re}[\vec{E}]$ pattern on the $x y$-plane using a monopole antenna placed vertically and $8 \mathrm{~mm}$ above the metasurface and the mushroom structure, when the metasurface is illuminated by a normally incident $x$-polarized plane wave. Reproduced from [93] with permission. (e) The working principle of similar type of bi-functional metasurface operating for visible light realized in [94]. The top panel shows the schematic of the unit cell consisting of an Ag nanobrick (grey) on top of a spacer (green) and Ag substrate. Reproduced from [94] with permission. SPPs, surface plasmon polaritons. 
In designing these devises, people noticed that an arbitrary anisotropic meta-atom might exhibit undesired polarization cross-talking (i.e., varying one parameter of the meta-atom can influence its EM responses for both polarizations). Such an effect hinders the fast designs of meta-devices with complex functionalities, which typically require 2D parameter searching to realize the desired phase profiles. To overcome such cross-talking and to enlarge the working bandwidth, Xu et al. proposed a new type of anisotropic meta-atom [95] which consists of multilayers of planar resonators coupled with a metallic ground plane (Figure 5a). A crucial improvement is to add a wire loop to surround each planar resonator (see Figure 5a), which can significantly degrade the polarization cross-talking due to the screening effect [95].
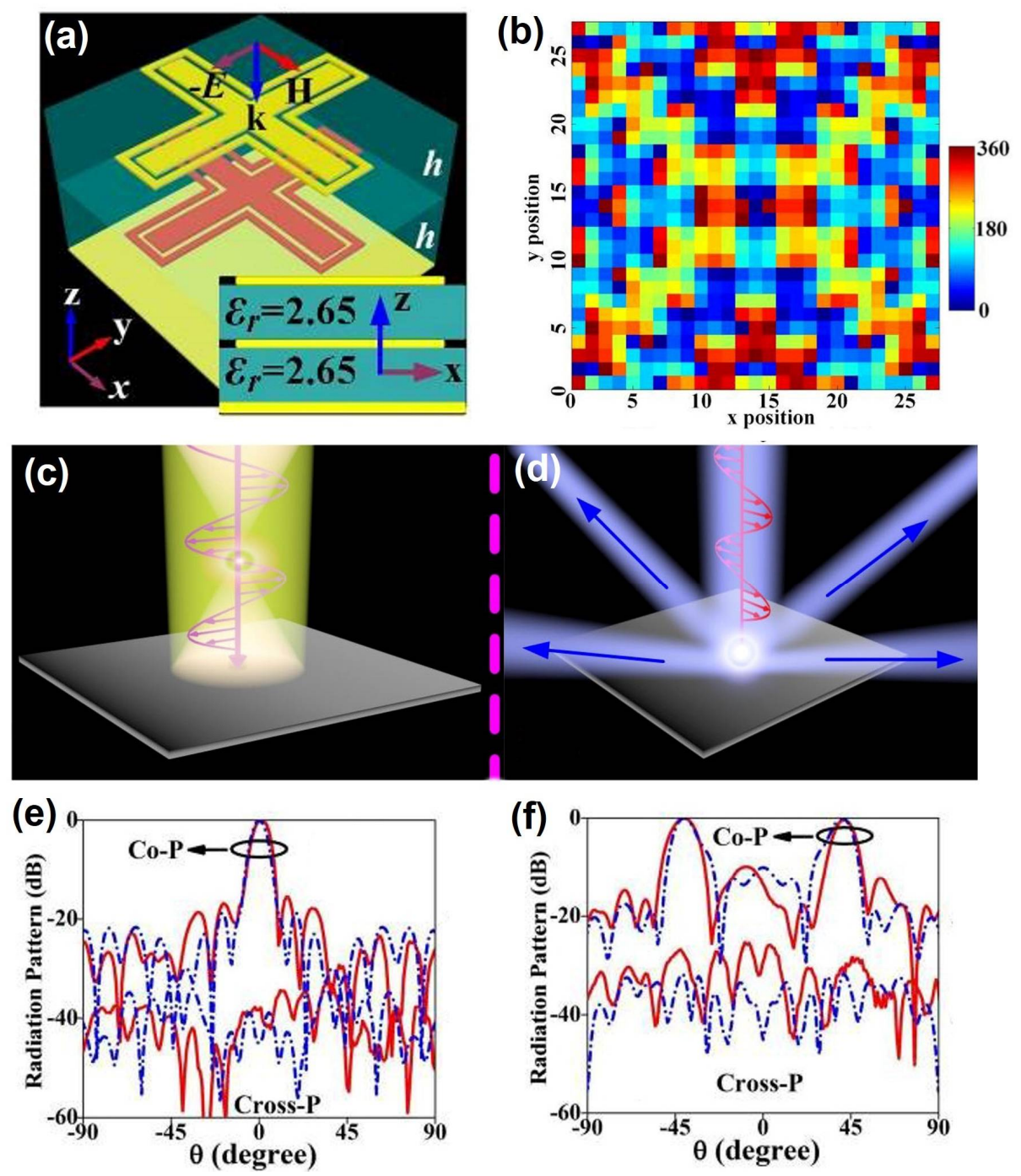

Figure 5. (a) Topology of the dual-layer anisotropic meta-atoms using composite cross bar and cross loop. The meta-atom contains two identical composite metallic resonators and a continuous metal plate (yellow) separated by two dielectric spacers (blue). (b) Phase distribution required on the metasurface to achieve quad-beam emissions. Schematics of a bifunctional metasurface which behaves as (c) a lens or (d) a beam splitter to generate quad large-angle pencil beams when excited by incident waves with polarizations $E / / x$ (yellow light) and $E / / y$ (blue light), respectively. (e,f) Simulated (red lines) and measured (blue dashed lines) radiation patterns on $x-z$ plane for $x$ polarization and $y$ polarization, demonstrating the bi-functionality possessed by the fabricated device. Reproduced from [95] with permission. 
Based on such meta-atoms, the authors successfully realized two bifunctional metasurfaces combining complex wave-control functionalities [95]. One device is shown in Figure 5 which can achieve the functionalities of focusing (Figure $5 \mathrm{c}$ ) and large-angle multi-beam emissions (Figure $5 \mathrm{~d}$ ) under external excitations with two polarizations. While the phase distribution responsible for the focusing functionality is easy to obtain (Equation (5)), that for another one needs to be carefully determined (see Figure 5b). The structural parameters of all meta-atoms can be quickly determined from two phase profiles, thanks to the low polarization cross talking property of the meta-atoms. Measured radiation patterns (Figure 5e,f) demonstrated clearly the bi-functionalities possessed by the fabricated device. Another device realized in [95] can deflect beam anomalously and achieve small-angle multi-beam emissions for two polarizations. With such a unique meta-atom structure at hand, people can realize other multifunctional meta-devices exhibiting powerful and complex wave-manipulation functionalities.

\subsection{Multifunctional Transmissive Metasurfaces Combining Distinct Functionalities}

Compared to reflective devices, high-efficiency multifunctional meta-devices in transmission geometry are much more difficult to realize, simply because more channels (transmission and reflection ones) exist in this geometry and thus Equation (2) is difficult to meet. People utilized the concept of Huygens' surface to construct transmission-mode metasurfaces with high efficiencies, but such devices usually require complex 3D non-flat meta-atoms with both electric and magnetic resonators incorporated $[24,26]$. Due to the complexities in meta-atom design, multifunctional meta-devices realized based on such a scheme are rarely seen, except a transmissive functionality-tunable device recently realized with controllable active elements incorporated [96].

Meanwhile, meta-atoms in multilayer geometry (with deep-subwavelength total thicknesses) are found as alternative candidates to construct high-efficiency transmission-mode metasurfaces. Many functional meta-devices are fabricated using such kind of meta-atoms [27,49], generating interesting effects such as high-efficiency SPP excitations [31] and photonic spin-Hall effect [54]. Straightforwardly, there appear recent efforts on using such meta-atoms to construct transmissive multifunctional meta-devices with very high efficiencies.

Cai et al. proposed to use a four-layer structure, with each layer containing a metallic mesh coupled with a metal patch [93], as the basic meta-atom to construct transmissive bifunctional meta-devices (Figure 6a). Each single layer exhibits perfect EM transmission at a particular frequency due to the interaction between the patch resonator and the opaque mesh. Mutual interactions between different layers can significantly enlarge the transparency window and the transmission-phase variation range (see Figure $6 \mathrm{~b}$ ). With such a high-performance structure, the authors designed and fabricated a transmissive metasurface (see Figure 6a for sample picture) with meta-atoms carefully adjusted to yield transmission phases $\left(\varphi_{x x}\right.$ and $\left.\varphi_{y y}\right)$ satisfying Equations (5) and (6). Experimental results shown in Figure 6e,f indicate that the device can focus $y$-polarized PW to a point and can refract $x$-polarized PW anomalously, illustrating the desired bi-functionality. The achieved working efficiency is $72 \%$, much higher than those of other transmissive metasurfaces [25-27,49]. 

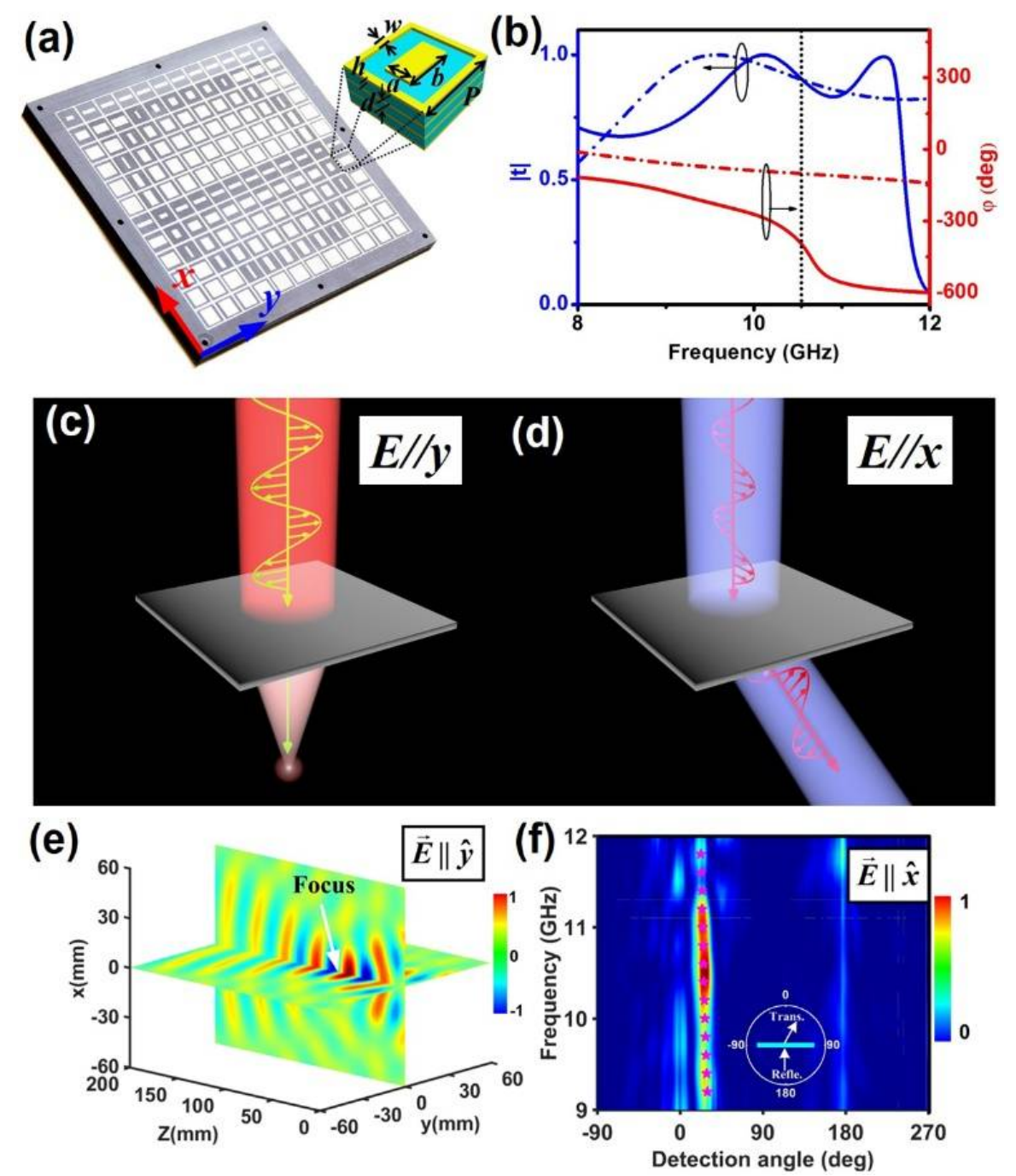

Figure 6. (a) Photograph of a microwave transmissive bifunctional metasurface. Inset illustrates a typical meta-atom composed by four metallic layers (yellow) separated by three F4B spacers (blue). (b) Transmission amplitude (blue lines) and phase (red lines) for a periodic metasurface constructed by the 4-layer meta-atom, under the excitations of $y$-polarized (solid lines) and $x$-polarized (dotted lines) incident waves, respectively. Schematics and working principles of a transmissive bifunctional metasurfaces, which behaves as (c) a focusing lens and (d) a beam deflector when excited by incident waves with polarizations $E / / y$ and $E / / x$, respectively. (e) Measured $\operatorname{Re}[\vec{E}]$ distributions on both $x_{\mathrm{o} z}$ and $y \mathrm{o} z$ planes as the metasurface is illuminated by a normally-incident $y$-polarized plane wave. (f) Measured scattered wave intensity as function of frequency and detection angle when the metasurface is illuminated by $x$-polarized normally incident plane waves. Reproduced from [93] with permission. 


\subsection{Multifunctional Metasurfaces for Full-Space Manipulation of EM Waves}

The multifunctional metasurfaces introduced in the previous sections, whether working in reflection or transmission geometries, leave half of the EM space completely unexplored. It is highly desired to expand the wave-manipulation capabilities of metasurfaces to the full EM space, offering the metasurfaces independently controlled functionalities at their two different sides. In this section, we introduce recent efforts to design metasurfaces that can manipulate the wave fronts of EM waves in the full EM space with very high efficiencies [97].

The key step in this new strategy is to design a collection of meta-atoms which are perfectly transparent or reflective for incident waves polarized along two orthogonal directions. As shown in the inset to Figure 7a, the meta-atom consists of four metallic layers separated by three dielectric spacers. A crucial difference of present meta-atom with a usual transmissive one (Figure 6a) is that here the $x$-orientated metallic stripes of the bottom two layers are continuous ones, which can ensure a high reflection for $x$-polarized waves. Meanwhile, the reflection-phase $\varphi_{x x}^{r}(\mathrm{x})$ for this polarization can vary from $-180^{\circ}$ to $180^{\circ}$ (Figure $7 \mathrm{~b}$ ) as frequency changes. For the $y$-polarization, however, the coupling between different layers creates a wideband transparent window with a controllable transmission phase, which can again cover the whole $360^{\circ}$ range (Figure $7 \mathrm{c}$ ).

Based on such unique meta-atoms, the authors successfully design several bi-functional meta-devices combining similar or distinct functionalities to manipulate EM waves at different sides of the metasurfaces. For instance, the authors fabricated a bifunctional metasurface, which can anomalously reflect $x$-polarized incident wave to the reflection side (Figure $7 \mathrm{~d}$ ) and focusing the $y$-polarized incident wave to a focal point at the transmission side (Figure 7e). As shown in Figure $7 f, g$, microwave experiments perfectly demonstrated the desired bi-functionalities of the fabricated meta-device. Both functionalities exhibit very high efficiencies (in the range of $85-91 \%$ ) and the total thickness of the device is only $\sim \lambda / 8$ [97].

Such a strategy has also been extended to design bi-functional PB metasurfaces that can manipulate the wave-fronts of circularly-polarized waves in the full space [89]. The key step is again to find a high-performance PB meta-atom exhibiting unique helicity-dependent transmission/reflection responses. Assisted by an elegant Jones' matrix analysis, Cai et al. were able to find a particular PB meta-atom (see Figure 8a) that can (nearly) perfectly reflect RCP light to RCP light at the reflection side (Figure 8b), and (nearly) transmit LCP light to RCP light at the transmission side (Figure 8c). Such unique polarization-conversion properties make the designed meta-atom ideal candidate to construct bifunctional PB meta-devices for controlling CP lights in the full space [54]. One can simply rotate the meta-atoms with appropriate angles to realize the desired phase distributions to achieve certain functionalities. The authors used such a meta-atom to fabricate a bifunctional meta-lens, and experimentally demonstrated that it behaves as a transmission-mode focusing lens for LCP wave (Figure 8d,f), but changes to a reflection-mode diverging lens for RCP wave (Figure 8e,g). Other bi-functional meta-devices can also be realized. However, one should note that the bi-functional meta-devices realized by such a scheme can only exhibit the same type of wave-manipulation functionalities for two polarizations, since the two phase distributions are intrinsically linked with each other. 
(a)

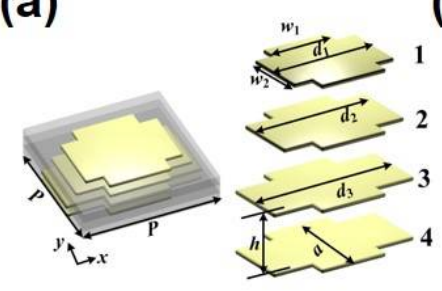

(b)

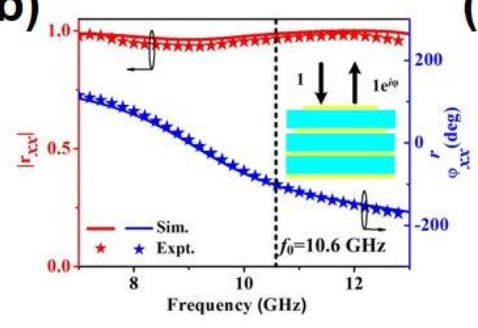

(c)

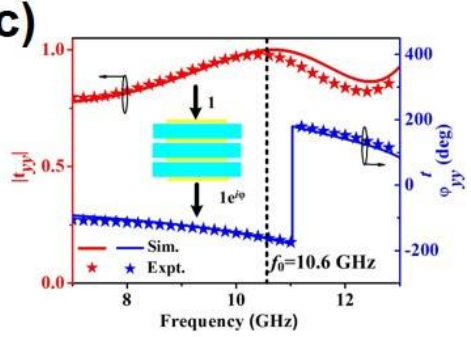

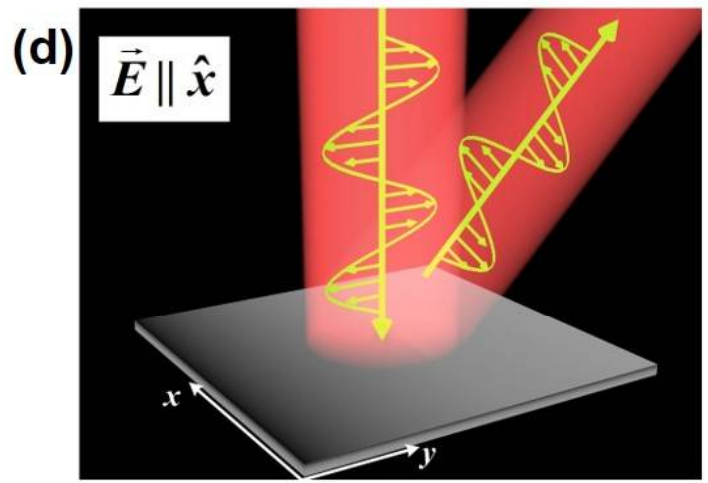

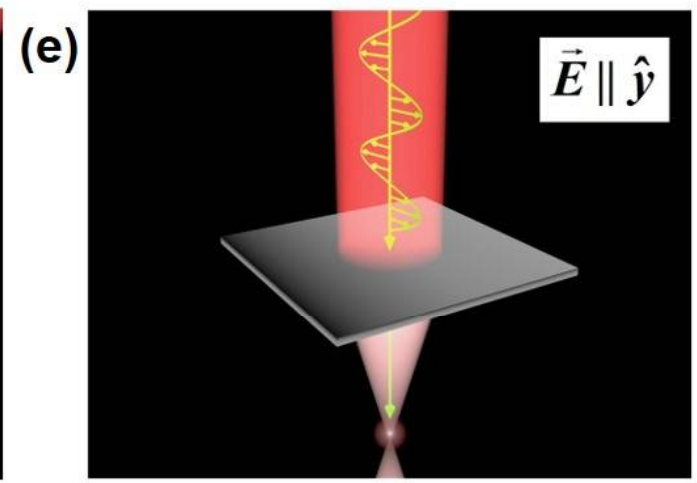

(f)

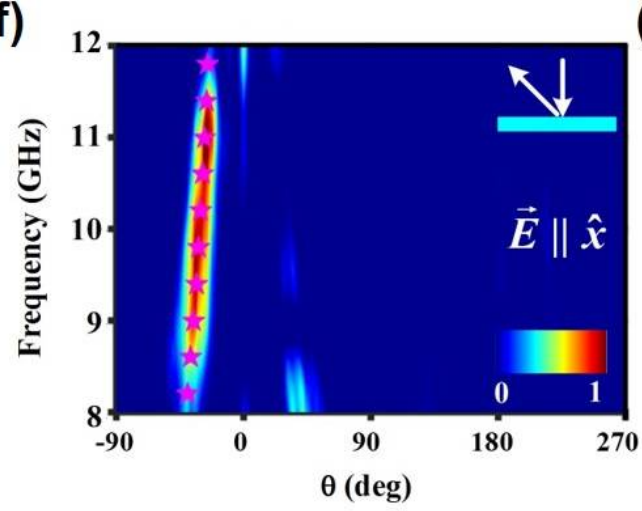

(g)

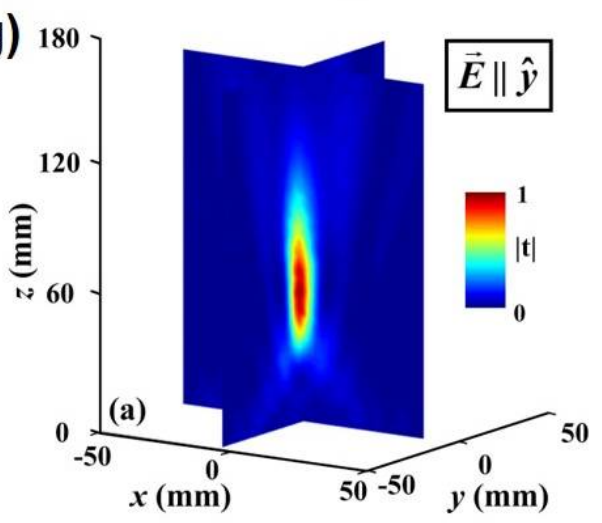

Figure 7. (a) Schematics of a 4-layer meta-atom composed by four metallic layers separated by three spacers. Measured and Finite-Difference Time-Domain (FDTD) simulated amplitude-phase spectra of reflection (b) and transmission (c) for a periodic metasurface made by the meta-atom given in (a), under excitations with different polarizations. Schematics of a bi-functional metasurface which behaves as (d) a reflective beam deflector and (e) a transmissive lens under excitations of $x$ - and $y$-polarized waves, respectively. Measured scattered field intensity versus frequency and detecting angle at reflection sides (f) of the metasurface shined by $x$-polarized microwaves and electric field distributions on both $x \mathrm{o} z$ and yoz planes at transmission sides ( $\mathrm{g}$ ) of the metasurface shined by $y$-polarized microwaves. Reproduced from [97] with permission. 
(a)

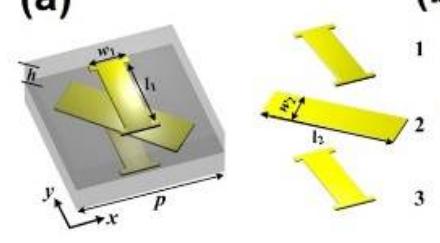

(b)

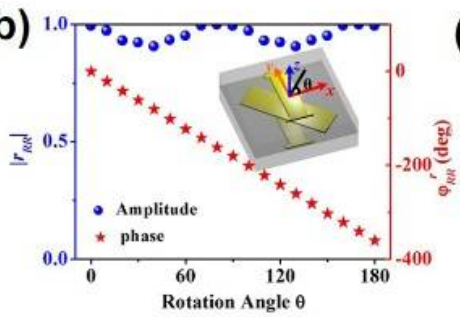

(c)

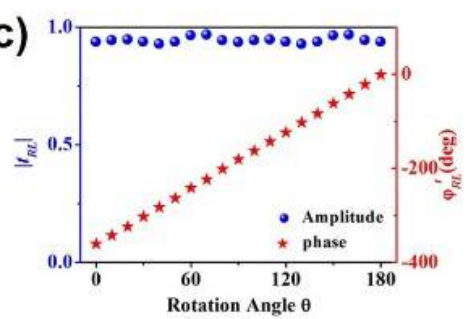

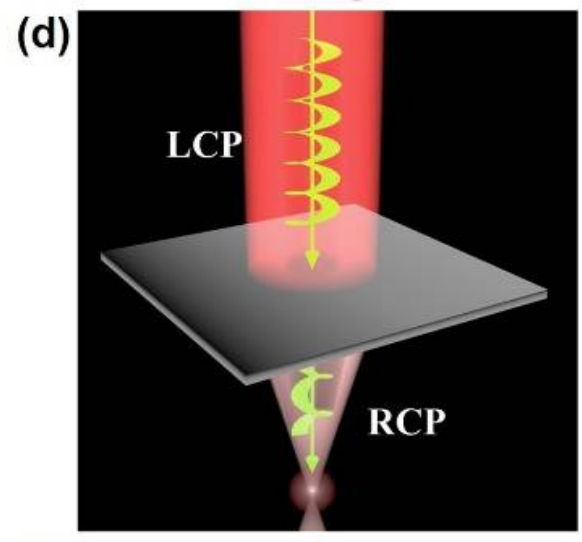

(f)

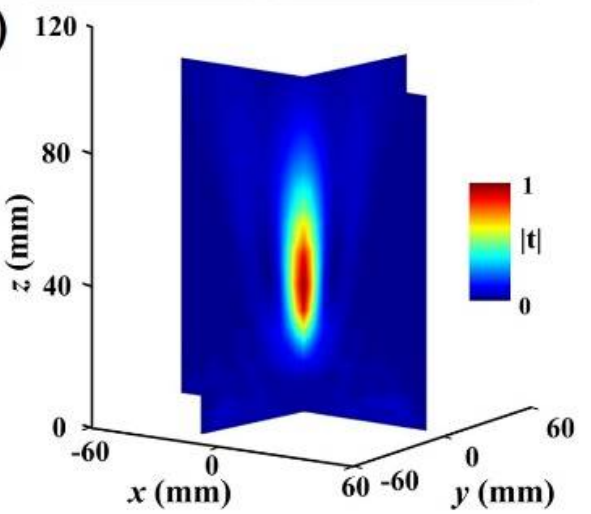

(e)

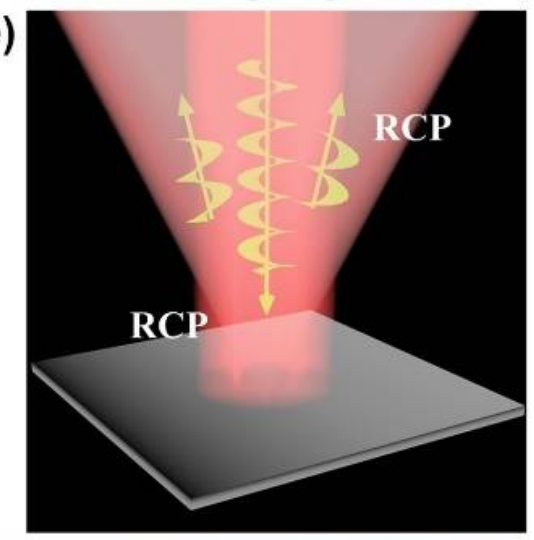

(g)

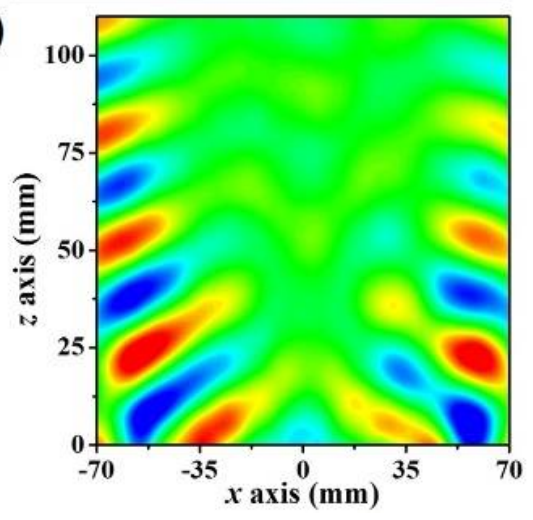

Figure 8. (a) Topology of a meta-atom consisting of three metallic layers (yellow) separated by two diecltric spacers (gray). (b,c) Amplitude/phase (Blue circle/red star) responses of the meta-atom rotated with certain angles when illuminated by normally incident (b) RCP and (c) LCP waves. Schematics of a bifunctional metasurface which behaves as a transmissive focusing lens (d) and a reflective diverging lens (e) under excitations of LCP and RCP waves, respectively. (f) Measured $\left|E_{x}\right|^{2}$ distributions on both $x_{\mathrm{o}} z$ and $y \mathrm{o} z$ planes at the transmission side when the meta-device is illuminated by a normally incident LCP wave. (g) Measured $\operatorname{Re}\left(E_{x}\right)$ distributions on the $x \mathrm{o} z$ plane at the reflection side of the metasurface under excitation of a normally-incident RCP wave. Reproduced from [89] with permission.

\section{Conclusions and Discussion}

In summary, we presented a concise review on the development of multifunctional metasurfaces, focusing on the physical mechanisms and practical meta-devices realizations. The design strategies include a simple "merging" concept and more sophisticated ones relying on single anisotropic meta-atoms, and the realized meta-devices can achieve two or more wave-manipulation functionalities at frequencies ranging from microwave to the visible. In reviewing the development of this field, we find that the meta-atom design is of particular importance, since any new type of high-performance multifunctional meta-atom can surely stimulate a series of meta-devices with diversified functionalities. We already see many interesting new designs in the field (for example, the polarization-dependent full-space meta-atom) which help people realize useful functional devices with more flexibilities in controlling EM waves. However, we also noticed that this field is far from mature, since many new 
ideas are only realized in the microwave regime, leaving plenty of rooms for researchers working at more challenging frequency domains. We hope that this review can serve as a useful guide to help researchers quickly jump into this field, eventually pushing these conceptual laboratory-level protocol devices to integration-optics platforms.

Before concluding this review, we would like to discuss more on the differences in the design of meta-atoms for microwave, Infrared Radiation (IR), or optical frequencies. Given the different behavior of materials in these frequency ranges, the geometry of the meta-atoms needed to achieve a chosen functionality are necessarily different. For example, whereas in the microwave regime metals behave as perfect electric conductors, the metallic losses can be very significant at IR and optical frequencies. As a result, while meta-atoms with multilayer metallic structures are widely used in designing high-efficiency transmissive multifunctional metasurfaces in low-frequency domains, these meta-atom structures are difficult to be used directly at high frequencies because of the fabrication challenges and the material losses. Instead, simple metallic structures (say, metal bars) are currently used in designing transmission-mode multifunctional meta-devices at high frequencies, although the realized meta-devices suffer from low-efficiency issues. Very recently, all-dielectric metasurfaces began to appear, which seems to be a very promising route to overcome such issue for transmissive multifunctional meta-devices at high frequencies. Along with the fast development in nanofabrication technologies, we expect that more excellent works can appear in this field, generating meta-devices which can eventually be used in practice.

Acknowledgments: This work is supported by the National Natural Science Foundation of China (grant no. 11604167 , no. 11474057 , no. 11404063 , no. 11734007 , no. 11674068 , and no. 61501499 ), National Basic Research Program of China (grant no. 2017YFA0303504), K. C. Wong Magna Fund in Ningbo University and the Shanghai Science and Technology Committee (grant no. 16ZR1445200 and no. 16JC1403100).

Author Contributions: Tong Cai, He-Xiu Xu, Qiong He and Shulin Sun contributed related materials; Shiwei Tang and Lei Zhou wrote the paper.

Conflicts of Interest: The authors declare no conflict of interest.

\section{References}

1. Shelby, R.A.; Smith, D.R.; Schultz, S. Experimental verification of a negative index of refraction. Science 2001, 292, 77-79. [CrossRef] [PubMed]

2. Veselago, V.G. The electrodynamics of substances with simultaneously negative values of epsilon and mu. Soviet Phys. Uspekhi 1968, 10, 509-514. [CrossRef]

3. Pendry, J.B. Negative refraction makes a perfect lens. Phys. Rev. Lett. 2000, 85, 3966. [CrossRef] [PubMed]

4. Valentine, J.; Zhang, S.; Zentgraf, T.; Ulinavila, E.; Genov, D.A.; Bartal, G.; Zhang, X. Three-dimensional optical metamaterial with a negative refractive index. Nature 2008, 455, 376. [CrossRef] [PubMed]

5. Liu, Z.; Lee, H.; Xiong, Y.; Sun, C.; Zhang, X. Far-field optical hyperlens magnifying sub-diffraction-limited objects. Science 2007, 315, 1686. [CrossRef] [PubMed]

6. Fang, N.; Lee, H.; Sun, C.; Zhang, X. Sub-Diffraction-Limited Optical Imaging with a Silver Superlens. Science 2005, 308, 534-537. [CrossRef] [PubMed]

7. Pendry, J.B.; Schurig, D.; Smith, D.R. Controlling electromagnetic fields. Science 2006, 312, 1780-1782. [CrossRef] [PubMed]

8. Liu, R.; Ji, C.; Mock, J.J.; Chin, J.Y.; Cui, T.J.; Smith, D.R. Broadband ground-plane cloak. Science 2009, 323, 366-369. [CrossRef] [PubMed]

9. Ma, H.F.; Cui, T.J. Three-dimensional broadband ground-plane cloak made of metamaterials. Nat. Commun. 2010, 1, 21. [CrossRef] [PubMed]

10. Sun, W.; He, Q.; Hao, J.; Zhou, L. A transparent metamaterial to manipulate electromagnetic wave polarizations. Opt. Lett. 2011, 36, 927-929. [CrossRef] [PubMed]

11. Ma, S.; Wang, X.; Luo, W.; Sun, S.; Zhang, Y.; He, Q.; Zhou, L. Ultra-wide band reflective metamaterial wave plates for terahertz waves. Europhys. Lett. 2017, 117, 37007. [CrossRef]

12. Hao, J.; Ren, Q.; An, Z.; Huang, X.; Chen, Z.; Qiu, M.; Zhou, L. Optical metamaterial for polarization control. Phys. Rev. A 2009, 80, 23807. [CrossRef] 
13. Hao, J.; Yuan, Y.; Ran, L.; Jiang, T.; Kong, J.A.; Chan, C.T.; Zhou, L. Manipulating Electromagnetic Wave Polarizations by Anisotropic Metamaterials. Phys. Rev. Lett. 2007, 99, 63908. [CrossRef] [PubMed]

14. Hao, J.; Wang, J.; Liu, X.; Padilla, W.J.; Zhou, L.; Qiu, M. High performance optical absorber based on a plasmonic metamaterial. Appl. Phys. Lett. 2010, 96, 251104. [CrossRef]

15. Song, Z.; He, Q.; Xiao, S.; Zhou, L. Making a continuous metal film transparent via scattering cancellations. Appl. Phys. Lett. 2012, 101, 5822. [CrossRef]

16. Andryieuski, A.; Lavrinenko, A.V.; Gritti, C.; Zhou, L.; Zalkovskij, M.; Jepsen, P.U.; He, Q.; Malureanu, R.; Song, Z. A new method for obtaining transparent electrodes. Opt. Express 2012, 20, 22770-22782.

17. Enoch, S.; Tayeb, G.; Sabouroux, P.; Guérin, N.; Vincent, P. A metamaterial for directive emission. Phys. Rev. Lett. 2002, 89, 213902. [CrossRef] [PubMed]

18. Jiang, Z.H.; Wu, Q.; Werner, D.H. Demonstration of enhanced broadband unidirectional electromagnetic radiation enabled by a subwavelength profile leaky anisotropic zero-index metamaterial coating. Phys. Rev. B 2012, 86, 125131. [CrossRef]

19. Jeon, S.; Menard, E.; Park, J.; Maria, J.; Meitl, M.; Zaumseil, J.; Rogers, J. Three-dimensional nanofabrication with rubber stamps and conformable photomasks. Adv. Mater. 2004, 16, 1369-1373. [CrossRef]

20. Rill, M.; Plet, C.; Thiel, M.; Wegener, M.; Freymann, G.V.; Linden, S. Photonic Metamaterials by Direct Laser Writing and Silver Chemical Vapor Deposition. Nat. Mater. 2008, 7, 543-546. [CrossRef] [PubMed]

21. Ni, X.; Emani, N.K.; Kildishev, A.V.; Boltasseva, A.; Shalaev, V.M. Broadband light bending with plasmonic nanoantennas. Science 2012, 335, 427. [CrossRef] [PubMed]

22. Pors, A.; Albrektsen, O.; Radko, I.P.; Bozhevolnyi, S.I. Gap plasmon-based metasurfaces for total control of reflected light. Sci. Rep. 2013, 3, 2155. [CrossRef] [PubMed]

23. Sun, S.; Yang, K.Y.; Wang, C.M.; Juan, T.K.; Chen, W.T.; Liao, C.Y.; He, Q.; Xiao, S.; Kung, W.T.; Guo, G.Y. High-Efficiency Broadband Anomalous Reflection by Gradient Meta-Surfaces. Nano Lett. 2012, 12, 6223-6229. [CrossRef] [PubMed]

24. Pfeiffer, C.; Grbic, A. Metamaterial Huygens' surfaces: tailoring wave fronts with reflectionless sheets. Phys. Rev. Lett. 2013, 110, 197401. [CrossRef] [PubMed]

25. Luo, J.; Yu, H.; Song, M.; Zhang, Z. Highly efficient wavefront manipulation in terahertz based on plasmonic gradient metasurfaces. Opt. Lett. 2014, 39, 2229-2231. [CrossRef] [PubMed]

26. Pfeiffer, C.; Emani, N.K.; Shaltout, A.M.; Boltasseva, A.; Shalaev, V.M.; Grbic, A. Efficient light bending with isotropic metamaterial Huygens' surfaces. Nano Lett. 2014, 14, 2491. [CrossRef] [PubMed]

27. Wei, Z.; Cao, Y.; Su, X.; Gong, Z.; Long, Y.; Li, H. Highly efficient beam steering with a transparent metasurface. Opt. Express 2013, 21, 10739. [CrossRef] [PubMed]

28. Yu, N.; Genevet, P.; Kats, M.A.; Aieta, F.; Tetienne, J.P.; Capasso, F.; Gaburro, Z. Light Propagation with Phase Discontinuities Reflection and Refraction. Science 2011, 334, 333. [CrossRef] [PubMed]

29. Miao, Z.; Wu, Q.; Li, X.; He, Q.; Ding, K.; An, Z.; Zhang, Y.; Zhou, L. Widely Tunable Terahertz Phase Modulation with Gate-Controlled Graphene Metasurfaces. Phys. Rev. X 2015, 5, 41027. [CrossRef]

30. Sun, S.; He, Q.; Xiao, S.; Xu, Q.; Li, X.; Zhou, L. Gradient-index meta-surfaces as a bridge linking propagating waves and surface waves. Nat. Mater. 2012, 11, 426-431. [CrossRef] [PubMed]

31. Sun, W.; He, Q.; Sun, S.; Zhou, L. High-efficiency surface plasmon meta-couplers: concept and microwave-regime realizations. Light Sci. Appl. 2016, 5, e16003. [CrossRef]

32. Lockyear, M.J.; Hibbins, A.P.; Sambles, J.R. Microwave surface-plasmon-like modes on thin metamaterials. Phys. Rev. Lett. 2009, 102, 73901. [CrossRef] [PubMed]

33. Xiong, X.; Hu, Y.S.; Jiang, S.C.; Hu, Y.H.; Fan, R.H.; Ma, G.B.; Shu, D.J.; Peng, R.W.; Wang, M. Metallic stereostructured layer: An approach for broadband polarization state manipulation. Appl. Phys. Lett. 2014, 105, 1304. [CrossRef]

34. Ding, F.; Wang, Z.; He, S.; Shalaev, V.M.; Kildishev, A.V. Broadband High-Efficiency Half-Wave Plate: A Super-Cell Based Plasmonic Metasurface Approach. ACS Nano 2015, 9, 4111-4119. [CrossRef] [PubMed]

35. Yang, Y.; Wang, W.; Moitra, P.; Kravchenko, I.I.; Briggs, D.P.; Valentine, J. Dielectric meta-reflectarray for broadband linear polarization conversion and optical vortex generation. Nano Lett. 2014, 14, 1394-1399. [CrossRef] [PubMed]

36. Jiang, S. Controlling the Polarization State of Light with a Dispersion-Free Metastructure. Phys. Rev. X 2014, 4, 21026. [CrossRef] 
37. Pfeiffer, C.; Grbic, A. Bianisotropic Metasurfaces for Optimal Polarization Control: Analysis and Synthesis. Phys. Rev. Appl. 2014, 2, 44011. [CrossRef]

38. Pfeiffer, C.; Zhang, C.; Ray, V.; Guo, L.J.; Grbic, A. High performance bianisotropic metasurfaces: asymmetric transmission of light. Phys. Rev. Lett. 2014, 113, 23902. [CrossRef] [PubMed]

39. Lee, B.; Yun, H.; Sung, J.; Yun, J.G.; Kim, J.; Lee, K.; Kim, S.J.; Lee, Y. Broadband ultrathin circular polarizer at visible and near-infrared wavelengths using a non-resonant characteristic in helically stacked nano-gratings. Opt. Express 2017, 25, 14260.

40. Li, X.; Xiao, S.; Cai, B.; He, Q.; Cui, T.J.; Zhou, L. Flat metasurfaces to focus electromagnetic waves in reflection geometry. Opt. Lett. 2012, 37, 4940. [CrossRef] [PubMed]

41. Aieta, F.; Kats, M.A.; Genevet, P.; Capasso, F. Multiwavelength achromatic metasurfaces by dispersive phase compensation. Science 2015, 347, 1342-1345. [CrossRef] [PubMed]

42. Ma, X.; Pu, M.; Li, X.; Huang, C.; Wang, Y.; Pan, W.; Zhao, B.; Cui, J.; Wang, C.; Zhao, Z.Y. A planar chiral meta-surface for optical vortex generation and focusing. Sci. Rep. 2015, 5, 10365. [CrossRef] [PubMed]

43. Huang, L.; Chen, X.; Mühlenbernd, H.; Zhang, H.; Chen, S.; Bai, B.; Tan, Q.; Jin, G.; Cheah, K.; Qiu, C. Three-dimensional optical holography using a plasmonic metasurface. Nat. Commun. 2013, 4, 2808. [CrossRef]

44. Zheng, G.; Mühlenbernd, H.; Kenney, M.; Li, G.; Zentgraf, T.; Zhang, S. Metasurface holograms reaching 80\% efficiency. Nat. Nanotechnol. 2015, 10, 308. [CrossRef] [PubMed]

45. Lee, G.Y.; Yoon, G.; Lee, S.Y.; Yun, H.; Cho, J.; Lee, K.; Kim, H.; Rho, J.; Lee, B. Complete amplitude and phase control of light using broadband holographic metasurfaces. Nanoscale 2018, 10, 4237-4245. [CrossRef] [PubMed]

46. Khorasaninejad, M.; Chen, W.T.; Devlin, R.C.; Oh, J.; Zhu, A.Y.; Capasso, F. Metalenses at visible wavelengths: Diffraction-limited focusing and subwavelength resolution imaging. Science 2016, 352, 1190. [CrossRef] [PubMed]

47. Arbabi, A.; Horie, Y.; Ball, A.J.; Bagheri, M.; Faraon, A. Subwavelength-thick lenses with high numerical apertures and large efficiency based on high-contrast transmitarrays. Nat. Commun. 2014, 6, 7069. [CrossRef] [PubMed]

48. Chen, X.; Chen, M.; Mehmood, M.Q.; Wen, D.; Yue, F.; Qiu, C.W.; Zhang, S. Longitudinal Multifoci Metalens for Circularly Polarized Light. Adv. Opt. Mater. 2015, 3, 1201-1206. [CrossRef]

49. Pfeiffer, C.; Grbic, A. Cascaded metasurfaces for complete phase and polarization control. Appl. Phys. Lett. 2013, 102, 231116. [CrossRef]

50. Ho, J.S.; Qiu, B.; Tanabe, Y.; Yeh, A.J.; Fan, S.; Poon, A.S.Y. Planar immersion lens with metasurfaces. Phys. Rev. B 2015, 91, 125145. [CrossRef]

51. Ju, Y.K.; Kim, H.; Kim, B.H.; Chang, T.; Lim, J.; Jin, H.M.; Mun, J.H.; Choi, Y.J.; Chung, K.; Shin, J. Highly tunable refractive index visible-light metasurface from block copolymer self-assembly. Nat. Commun. 2016, 7, 12911.

52. Park, J.; Kang, J.; Kim, S.J.; Liu, X.; Brongersma, M.L. Dynamic Reflection Phase and Polarization Control in Metasurfaces. Nano Lett. 2016, 17, 407. [CrossRef] [PubMed]

53. Shi, Y.; Fan, S. Dynamic non-reciprocal meta-surfaces with arbitrary phase reconfigurability based on photonic transition in meta-atoms. Appl. Phys. Lett. 2016, 108, 1232009-1232150. [CrossRef]

54. Luo, W.; Xiao, S.; He, Q.; Sun, S.; Zhou, L. Photonic Spin Hall Effect with Nearly 100\% Efficiency. Adv. Opt. Mater. 2015, 3, 1102-1108. [CrossRef]

55. Yin, X.; Ye, Z.; Rho, J.; Wang, Y.; Zhang, X. Photonic spin Hall effect at metasurfaces. Science 2013, 339, 1405-1407. [CrossRef] [PubMed]

56. Kildishev, A.; Shaltout, A.; Liu, J.; Shalaev, V. Photonic spin Hall effect in gap-plasmon metasurfaces for on-chip chiroptical spectroscopy. Optica 2015, 2, 860.

57. Yue, F.; Wen, D.; Zhang, C.; Gerardot, B.D.; Wang, W.; Zhang, S.; Chen, X. Multichannel Polarization-Controllable Superpositions of Orbital Angular Momentum States. Adv. Mater. 2017, 29, 1603838. [CrossRef] [PubMed]

58. Mehmood, M.Q.; Mei, S.; Hussain, S.; Huang, K.; Siew, S.Y.; Zhang, L.; Zhang, T.; Ling, X.; Liu, H.; Teng, J. Visible-Frequency Metasurface for Structuring and Spatially Multiplexing Optical Vortices. Adv. Mater. 2016, 28, 2533-2539. [CrossRef] [PubMed] 
59. Chen, W.T.; Yang, K.Y.; Wang, C.M.; Huang, Y.W.; Sun, G.; Chiang, I.; Liao, C.Y.; Hsu, W.L.; Lin, H.T.; Sun, S. High-Efficiency Broadband Meta-Hologram with Polarization-Controlled Dual Images. Nano Lett. 2014, 14, 225-230. [CrossRef] [PubMed]

60. Wan, X.; Shen, X.; Luo, Y.; Cui, T.J. Planar bifunctional Luneburg-fisheye lens made of an anisotropic metasurface. Laser Photonics Rev. 2014, 8, 757-765. [CrossRef]

61. Huang, Y.W.; Wei, T.C.; Tsai, W.Y.; Wu, P.C.; Wang, C.M.; Sun, G.; Tsai, D.P. Aluminum Plasmonic Multicolor Meta-Hologram. Nano Lett. 2015, 15, 3122. [CrossRef] [PubMed]

62. Wen, D.; Chen, S.; Yue, F.; Chan, K.; Chen, M.; Ardron, M.; Li, K.F.; Wong, P.W.H.; Cheah, K.W.; Pun, E.Y.B. Metasurface Device with Helicity-Dependent Functionality. Adv. Opt. Mater. 2016, 4, 321-327. [CrossRef]

63. Wen, D.; Yue, F.; Li, G.; Zheng, G.; Chan, K.; Chen, S.; Chen, M.; Li, K.F.; Wong, P.W.H.; Cheah, K.W. Helicity multiplexed broadband metasurface holograms. Nat. Commun. 2015, 6, 8241. [CrossRef] [PubMed]

64. Huang, L.; Mühlenbernd, H.; Li, X.; Song, X.; Bai, B.; Wang, Y.; Zentgraf, T. Broadband hybrid holographic multiplexing with geometric metasurfaces. Adv. Mater. 2015, 27, 6444-6449. [CrossRef] [PubMed]

65. Wen, D.; Yue, F.; Ardron, M.; Chen, X. Multifunctional metasurface lens for imaging and Fourier transform. Sci. Rep. 2016, 6, 27628. [CrossRef] [PubMed]

66. Liu, S.; Zhang, L.; Yang, Q.L.; Xu, Q.; Yang, Y.; Noor, A.; Zhang, Q.; Iqbal, S.; Wan, X.; Tian, Z.; et al. Frequency-Dependent Dual-Functional Coding Metasurfaces at Terahertz Frequencies. Adv. Opt. Mater. 2016, 4, 1965-1973. [CrossRef]

67. Wang, X.; Ding, J.; Zheng, B.; An, S.; Zhai, G.; Zhang, H. Simultaneous Realization of Anomalous Reflection and Transmission at Two Frequencies using Bi-functional Metasurfaces. Sci. Rep. 2018, 8, 1876. [CrossRef] [PubMed]

68. Stewart, J.W.; Akselrod, G.M.; Smith, D.R.; Mikkelsen, M.H. Toward Multispectral Imaging with Colloidal Metasurface Pixels. Adv. Mater. 2017, 29, 1602971. [CrossRef] [PubMed]

69. Veksler, D.; Maguid, E.; Shitrit, N.; Ozeri, D.; Kleiner, V.; Hasman, E. Multiple Wavefront Shaping by Metasurface Based on Mixed Random Antenna Groups. ACS Photonics 2015, 2, 661-667. [CrossRef]

70. Xiang, N.; Cheng, Q.; Chen, H.B.; Zhao, J.; Jiang, W.X.; Ma, H.F.; Cui, T.J. Bifunctional metasurface for electromagnetic cloaking and illusion. Appl. Phys. Express 2015, 8, 92601. [CrossRef]

71. Chen, X.; Huang, L.; Mühlenbernd, H.; Li, G.; Bai, B.; Tan, Q.; Jin, G.; Qiu, C.; Zhang, S.; Zentgraf, T. Dual-polarity plasmonic metalens for visible light. Nat. Commun. 2012, 3, 1198. [CrossRef] [PubMed]

72. Pors, A.; Nielsen, M.G.; Bernardin, T.; Weeber, J.; Bozhevolnyi, S.I. Efficient unidirectional polarization-controlled excitation of surface plasmon polaritons. Light Sci. Appl. 2014, 3, e197. [CrossRef]

73. Hou, H.; Wang, G.; Li, H.; Guo, W.; Li, T. Highly efficient multifunctional metasurface for high-gain lens antenna application. Appl. Phys. A 2017, 123, 460. [CrossRef]

74. Zhang, C.; Yue, F.; Wen, D.; Chen, M.; Zhang, Z.; Wang, W.; Chen, X. Multichannel metasurface for simultaneous control of holograms and twisted light beams. ACS Photonics 2017, 4, 1906-1912. [CrossRef]

75. Li, Y.; Li, X.; Chen, L.; Pu, M.; Jin, J.; Hong, M.; Luo, X. Orbital angular momentum multiplexing and demultiplexing by a single metasurface. Adv. Opt. Mater. 2017, 5, 1600502. [CrossRef]

76. Chen, X.; Huang, L.; Mühlenbernd, H.; Li, G.; Bai, B.; Tan, Q.; Jin, G.; Qiu, C.W.; Zentgraf, T.; Zhang, S. Reversible Three-Dimensional Focusing of Visible Light with Ultrathin Plasmonic Flat Lens. Adv. Opt. Mater. 2013, 1, 517-521. [CrossRef]

77. Wang, Z.; Jia, H.; Yao, K.; Cai, W.; Chen, H.; Liu, Y. Circular dichroism metamirrors with near-perfect extinction. ACS Photonics 2016, 3, 2096-2101. [CrossRef]

78. Cai, T.; Wang, G.M.; Zhang, X.F.; Liang, J.G.; Zhuang, Y.Q.; Liu, D.; Xu, H.X. Ultra-Thin Polarization Beam Splitter Using 2-D Transmissive Phase Gradient Metasurface. IEEE Trans. Antennas Propag. 2015, 63, 5629-5636. [CrossRef]

79. Ma, H.F.; Wang, G.Z.; Kong, G.S.; Cui, T.J. Independent controls of differently-polarized reflected waves by anisotropic metasurfaces. Sci. Rep. 2015, 5, 9605. [CrossRef] [PubMed]

80. Liu, S.; Cui, T.J.; Xu, Q.; Bao, D.; Du, L.; Wan, X.; Tang, W.X.; Ouyang, C.; Zhou, X.Y.; Yuan, H. Anisotropic coding metamaterials and their powerful manipulation of differently polarized terahertz waves. Light Sci. Appl. 2016, 5, e16076. [CrossRef]

81. Jia, S.L.; Wan, X.; Bao, D.; Zhao, Y.J.; Cui, T.J. Independent controls of orthogonally polarized transmitted waves using a Huygens metasurface. Laser Photonics Rev. 2015, 9, 545-553. [CrossRef] 
82. Zhu, A.Y.; Kuznetsov, A.I.; Luk Yanchuk, B.; Engheta, N.; Genevet, P. Traditional and emerging materials for optical metasurfaces. Nanophotonics 2016, 6, 452-471. [CrossRef]

83. Mosallaei, H.; Farmahinifarahani, M. Birefringent reflectarray metasurface for beam engineering in infrared. Opt. Lett. 2013, 38, 462.

84. Boroviks, S.; Deshpande, R.A.; Mortensen, N.A.; Bozhevolnyi, S.I. Multifunctional meta-mirror: polarization splitting and focusing. ACS Photonics 2017. [CrossRef]

85. Maguid, E.; Yulevich, I.; Veksler, D.; Kleiner, V.; Brongersma, M.L.; Hasman, E. Photonic spin-controlled multifunctional shared-aperture antenna array. Science 2016, 352, 1202. [CrossRef] [PubMed]

86. Lin, D.; Holsteen, A.L.; Maguid, E.; Wetzstein, G.; Kik, P.G.; Hasman, E.; Brongersma, M.L. Photonic Multitasking Interleaved Si Nanoantenna Phased Array. Nano Lett. 2016, 16, 7671. [CrossRef] [PubMed]

87. Song, E.Y.; Lee, G.Y.; Park, H.; Lee, K.; Kim, J.; Hong, J.; Kim, H.; Lee, B. Compact Generation of Airy Beams with C-Aperture Metasurface. Adv. Opt. Mater. 2017, 5, 1601028. [CrossRef]

88. Ling, X.; Zhou, X.; Shu, W.; Luo, H.; Wen, S. Realization of Tunable Photonic Spin Hall Effect by Tailoring the Pancharatnam-Berry Phase. Sci. Rep. 2014, 4, 5557. [CrossRef] [PubMed]

89. Cai, T.; Wang, G.; Xu, H.; Tang, S.; Li, H.; Liang, J.; Zhuang, Y. Bifunctional Pancharatnam-Berry metasurface with high-efficiency helicity-dependent transmissions and reflections. Ann. Phys. 2018, 530, 1700321. [CrossRef]

90. Cai, T.; Wang, G.; Xu, H.; Tang, S.; Liang, J. Polarization-independent broadband meta-surface for bifunctional antenna. Opt. Express 2016, 24, 22606-22615. [CrossRef] [PubMed]

91. Ma, S.; Xiao, S.; Zhou, L. Resonant modes in metal/insulator/metal metamaterials: An analytical study on near-field couplings. Phys. Rev. B 2016, 93, 45305. [CrossRef]

92. Qu, C.; Ma, S.; Hao, J.; Qiu, M.; Li, X.; Xiao, S.; Miao, Z.; Dai, N.; He, Q.; Sun, S. Tailor the Functionalities of Metasurfaces Based on a Complete Phase Diagram. Phys. Rev. Lett. 2015, 115, 235503. [CrossRef] [PubMed]

93. Cai, T.; Tang, S.; Wang, G.; Xu, H.; Sun, S.; He, Q.; Zhou, L. High-performance bifunctional metasurfaces in transmission and reflection geometries. Adv. Opt. Mater. 2017, 5, 1600506. [CrossRef]

94. Ding, F.; Deshpande, R.; Bozhevolnyi, S.I. Bifunctional Gap-Plasmon Metasurfaces for Visible Light: Polarization-Controlled Unidirectional Surface Plasmon Excitation and Beam Steering at Normal Incidence. Light Sci. Appl. 2018. [CrossRef]

95. Xu, H.X.; Tang, S.; Ling, X.; Luo, W.; Zhou, L. Flexible control of highly-directive emissions based on bifunctional metasurfaces with low polarization cross-talking. Ann. Phys. 2017, 529, 1700045. [CrossRef]

96. Chen, K.; Feng, Y.; Monticone, F.; Zhao, J.; Zhu, B.; Jiang, T.; Zhang, L.; Kim, Y.; Ding, X.; Zhang, S. A Reconfigurable Active Huygens' Metalens. Adv. Mater. 2017, 29, 1606422. [CrossRef] [PubMed]

97. Cai, T.; Wang, G.; Tang, S.; Xu, H.; Duan, J.; Guo, H.; Guan, F.; Sun, S.; He, Q.; Zhou, L. High-efficiency and full-space manipulation of electromagnetic wave fronts with metasurfaces. Phys. Rev. Appl. 2017, 8, 34033. [CrossRef]

(C) 2018 by the authors. Licensee MDPI, Basel, Switzerland. This article is an open access article distributed under the terms and conditions of the Creative Commons Attribution (CC BY) license (http://creativecommons.org/licenses/by/4.0/). 